\title{
Effectiveness of Using a Chief Complaint and Discharge Diagnosis Query in ESSENCE-FL to Identify Possible Tuberculosis Patients and Contacts in Hillsborough County, Florida
}

\author{
Michael Wiese ${ }^{\star 1}$, David Atrubin², Warren McDougle ${ }^{1}$ and Jylmarie Lewis ${ }^{1}$ \\ ${ }^{1}$ Epidemiology, FDOH-Hillsborough, Tampa, FL, USA; ${ }^{2}$ Florida Department of Health, Tallahassee, FL, USA
}

\section{Objective}

While syndromic surveillance systems were originally designed for the detection of outbreaks and clusters of illness, they have been found to be useful at identifying unreported conditions of public health importance. Within the Florida Department of Health in Hillsborough County (FDOH-Hillsborough), these conditions of public health importance have primarily focused on the reportable diseases and conditions that fall under the responsibility of the Epidemiology Program and have not included tuberculosis. A specific query has been developed to search for and identify possible tuberculosis patients and exposed contacts. This study is designed to determine the usefulness of specific-term chief complaint and discharge diagnosis (CCDD) queries in identifying tuberculosis patients and exposed contacts.

\section{Introduction}

The Electronic Surveillance System for the Early Notification of Community-based Epidemics in Florida (ESSENCE-FL) is a webbased application for use by public health professionals within the Florida Department of Health (FDOH). The main source of data for ESSENCE-FL is emergency department (ED) data. Ten hospitals in Hillsborough County, Florida send their data to the ESSENCE-FL server. ESSENCE-FL requires only a limited data set to be sent by the hospital which includes patient chief complaint (CC) and discharge diagnosis (DD). These fields can be searched individually, in separate queries, to identify possible records of interest. These two fields have been concatenated to create the single chief complaint and discharge diagnosis (CCDD) field, allowing both fields to be searched with a single query.

\section{Methods}

A free text query of the CCDD field for tuberculosis was developed. This query was written as: $\mathrm{TB}_{-}{ }^{\prime}$, or, $,{ }_{-} \mathrm{TB}, \mathrm{or},{ }^{\wedge}{ }^{\mathrm{TB}}{ }_{-}{ }^{\wedge}$,or, ${ }^{\wedge}$ tuberc $^{\wedge}$ The query was designed to detect ED visits in which TB or tuberculosis was written in the CC or DD fields. Epidemiologists with FDOH-Hillsborough ran the query daily. Using the query results, staff from the Tuberculosis Department followed up with hospitals to gather additional information on those patient visits. TB staff then determined if these ED visits were new or existing TB patients or exposed contacts of TB cases.

\section{Results}

For the 10 participating hospitals in Hillsborough County, ESSENCE-FL receives an average of 12,310 ED visits per week. A sample of the CCDD field that the query returned can be seen in Table 1 . Additionally, a graph of the number of ED visits, by week, that the query returned is shown in Graph 1.

\section{Conclusions}

This CCDD query field was developed in February of 2013 and has proven useful in detecting diseases of public importance that hospitals have failed to report. During a month-long trial period, this query returned a weekly average of $2.89 \mathrm{~TB}$ related visits per week from the 10 participating hospitals in Hillsborough County. Continued analysis will assess the usefulness of the query results. The benefit to public health is being evaluated based on the results of these follow up investigations. Results of this study will impact the specific query terms used statewide for ESSENCE-FL.

Table 1: A sample of the CCDD (chief complaint | discharge diagnosis) field that the query returned in July, 2013.

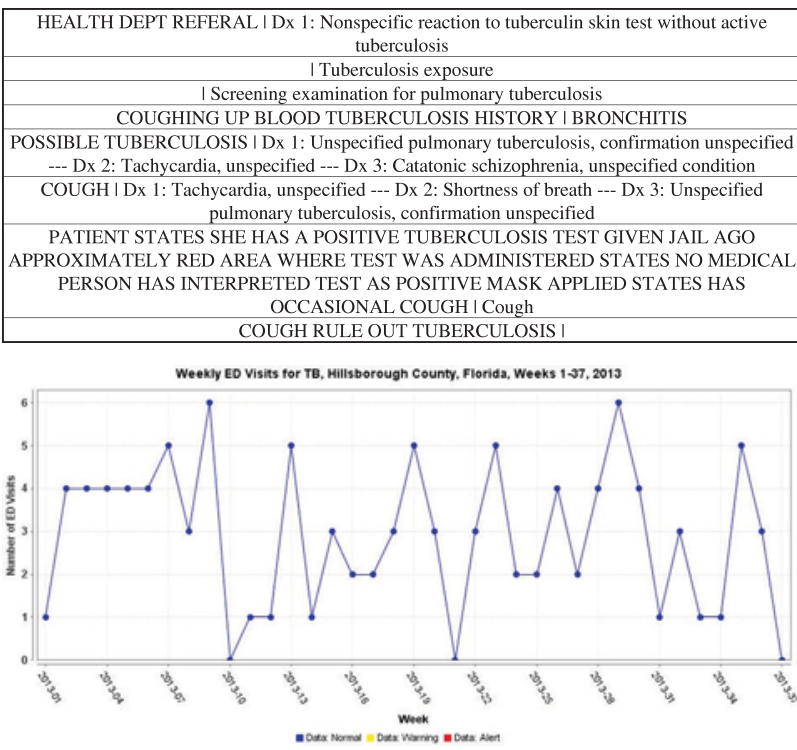

Graph 1: A retrospective graph of the number of ED visits, by week that the CCDD query returned.

\section{Keywords}

ESSENCE; Tuberculosis; TB; chief complaint; discharge diagnosis

\section{Acknowledgments}

Authors: Michael Wiese, MPH, CPH; David Atrubin, MPH; Jylmarie Lewis, MPH, CPH; Warren McDougle, MPH

*Michael Wiese

E-mail: Michael.Wiese@FLhealth.gov 